\title{
Performance Evaluation of a Hybrid Sensor and Vehicular Network to improve road safety
}

\author{
Carolina Tripp Barba \\ Telematic Engineering Dpt. \\ Polytechnic University of Catalonia \\ Barcelona, Spain \\ ctripp@entel.upc.edu
}

\author{
Karen Ornelas \\ Telematic Engineering Dpt. \\ Polytechnic University of Catalonia \\ Barcelona, Spain \\ kornelas@entel.upc.edu
}

\author{
Mónica Aguilar Igartua \\ Telematic Engineering Dpt. \\ Polytechnic University of Catalonia \\ Barcelona, Spain \\ monica.aguilar@entel.upc.edu
}

\begin{abstract}
In the last years, wireless networks have become a widely spread type of communication technology and also a challenging scientific area for new fields of research. Many contributions in ad hoc networks, such as WSNs (Wireless Sensor Networks) and VANETs (Vehicular Ad Hoc Networks), have been proposed. Nowadays, the huge amount of cars in transit has raised a big interest in vehicular communication technologies. A new type of network has been developed, named HSVN (Hybrid Sensor and Vehicular Network) in which WSNs and VANETs cooperate with the aim of improving road safety. Recent projects, such as CVIS [1] and COMeSafety [2], are focused on improving the road driving. This type of approaches will warn the driver and the co-pilot of any event occurred in the road ahead, such as traffic jam, accidents, bad weather, etc. This way, the number of traffic accidents may decrease and many lives might be saved. Besides, a better selection of non-congested roads will help to reduce pollution. In addition, other attractive services, such as downloading of multimedia services or Internet browsing, would be easily available through infrastructure along the roadside. Transportation in motorways will be easier, safer and more comfortable for passengers. In this paper a HSVN platform is presented, also a communications protocol between VANETs and WSNs is described and evaluated using the NCTUns [3] simulator.
\end{abstract}

\section{Categories and Subject Descriptors}

C.2.1 [Computer-Communication Networks]: Network Architecture and Design - Wireless Communication. C.2.5. [Computer-Communication Networks]: Network Protocols Protocol Verification. C.4 [Performance of Systems]: Design studies.

\section{General Terms \\ Design, Performance}

\section{Keywords}

WSN (Wireless Sensor Network), VANET (Vehicular ad hoc Network), HSVN (Hybrid Sensor and Vehicular Network).

Permission to make digital or hard copies of all or part of this work for personal or classroom use is granted without fee provided that copies are not made or distributed for profit or commercial advantage and that copies bear this notice and the full citation on the first page. To copy otherwise, or republish, to post on servers or to redistribute to lists, requires prior specific permission and/or a fee.

Conference'10, Month 1-2, 2010, City, State, Country.

Copyright 2010 ACM 1-58113-000-0/00/0010 ..\$10.00

\section{INTRODUCTION}

Research on short range technologies has been evolving very fast in the last years. In addition, ad hoc networks are receiving much attention due to the easy deployment they require. An ad hoc network [4] is formed by a group of nodes that communicate with each other by wireless interfaces, either with a fixed infrastructure or without any kind of infrastructure. In the framework under consideration, there are two kind of ad hoc networks: WSNs (Wireless Sensor Networks) [5] and VANETs (Vehicular Ad hoc Networks) [6, 7]. Both WSNs and VANETs are ad hoc networks which can operate without any infrastructure or centralized management. In such a case, the network organization is carried out by the nodes themselves. Every node is capable to work as a sender, destination or as a forwarding node. Nodes in WSNs are static, while nodes in VANETs can achieve very high speeds. This motion of the nodes produces frequent changes in network topology, so that the design of routing protocols able to adapt to the dynamic environment is a really challenging task.

WSNs consist of a small group of wireless devices able to gather information from their environment, such as temperature, humidity, movement, etc. This type of network allows fast deployment of their devices due to their small size and weight. Nonetheless, it has some restrictions in comparison to other ad hoc networks, such as their limited memory, scarce energy, small transmission range and low processing capacity. Those are the main issues to keep in mind when working with them.

A VANET can be considered as a particular type of MANET (Mobile Ad hoc Network) [4]. However, the main difference is the speed in nodes. This factor may produce quick changes in the network topology and thus turn out to short link lifetimes. In addition, vehicle's devices hardly have limitations regarding energy supply and can have a high processing power. By the end of 2010, the standard IEEE 802.11p [8,9] is expected to be released, and it will contribute to improve communication among vehicles and also between vehicles and RSU (Road Side Units). Also, vehicles are envisioned to carry multiple types of wireless transceivers to be able to communicate across more than one wireless data links; vehicles will be equipped with an OBU (On-Board Unit) which will manage the communication between different technologies (e.g. 2G/3G, WiMax, satellite). Besides, vehicles will easily get Internet connectivity through the closest available AP (Access Point) along the roadside.

A new approach has recently been proposed which merges both WSN and VANET networks. HSVNs (Hybrid Sensor and Vehicular Networks) consist in making WSNs and VANETs work jointly to constitute a communication framework to be used by vehicles in order to assist drivers to reduce road 
accidents, fatalities and injuries. Recently, new architectures have been proposed to offer robust, reliable and cost-effective approaches for HVSNs. HSVNs are introduced as a new concept of road sensor deployment, and they can be seen as a new kind of new generation network architecture. In a global perspective, weather events conditions, such as rain or ice, or the amount of traffic density in remote road segments can be monitorized by the cars. All the road information gathered by the cars will be stored in WSNs, which are deployed along the roadside, to be later spread among other passing vehicles. This way, VANETs enlarge their scope, since other passing vehicles can recover that information later. The purpose of HSVNs is that vehicles within the VANET can share information regarding climate conditions, traffic state and road safety, seeking to reduce the number of accidents. Thanks to this information interchange, users might drive safer along the routes.

\section{RELATED WORK}

Recently, different consortiums have been created in Europe [1, $2,10,11,12]$ which aim to make safer vehicles and roads. These consortiums are mainly integrated by car manufacturers, researchers and the European Commission. Among other projects, we highlight the following.

The CAR 2 CAR [10] communication consortium is a nonprofit industrial driven organization initiated by European vehicle manufacturers supported by equipment suppliers, research organizations and other partners. Their objective is to increase road safety and driving efficiency by means of cooperative intelligent transportation systems (ITS), vehicle to vehicle (V2V) and vehicle to infrastructure (V2I) communications.

The CVIS (Cooperative Vehicle-Infrastructure Systems) [1] project deals with intelligent co-operative systems that are based on $\mathrm{V} 2 \mathrm{~V}$ and $\mathrm{V} 2 \mathrm{I}$ communications to achieve improvements both in the efficiency of the transport systems and in the safety of all road users. The expected benefits stem from the increased information that is available of the vehicle and its environment. Those benefits include an increase in road network capacity, reduction of congestion and pollution, shorter and more predictable journey times, improved traffic safety for all road users, more efficient logistics, improved management and control of the road network (both urban and inter-urban), increased efficiency of the public transport systems and better and more efficient response to hazards, incidents and accidents.

CARLINK [11] seeks to develop intelligent service platforms for vehicles. The primary application of this project is to offer real time local weather information, transit reports and other broadcast applications. Vehicles are equipped with transceivers that are able to communicate with base stations and with other nodes of the ad hoc network. The goals of this project aim at improving car industry, telecommunication operators, private drivers, public transportation, truck traffic and other road users. New cars are foreseen to include new safety features and new kind of telecommunication services related to ITS, which will bring new kind of business opportunities to telecommunication operators.

The COMeSafety project [2] supports the eSafety Forum [12] with respect to all issues related to vehicle-to-vehicle and vehicle-to-infrastructure communications as the basis for cooperative intelligent road transport systems. Also, it provides an open integrating platform, aiming at the interests of all public and private road safety stakeholders to be represented. Consolidated results and interests are submitted to the European and worldwide standardization bodies.

INFOTRANSIT [13] has been developed by the RACC (Reial Automòbil Club de Catalunya) foundation which provides data to make a safer road. It consists in an Internet service based on different data sources that provide real time traffic, weather information, speed cameras location, traffic congestion, and accident location. Its interactive map is based on Google maps [14]. In the near future, drivers are expected to easily access to updated traffic information every moment during the trip in a cost-free fashion using the infrastructure along the roadside, e.g. RSUs. This traffic information will be provided by the Traffic Management Unit of each country. Drivers will easily be able to see the location of speed radars, short video streams from traffic cameras, or the location of any incident occurred in the roads.

Regarding HSVNs, several research $[15,16,17,18]$ studies have been made whose principal challenges are the architecture design. HSVNs need to include a reliable communication protocol between VANETs and WSNs, which have to interchange dynamic and static data from their respective nodes. Most of the studies make several assumptions such as GPS (Global Positioning System) [19] devices available in all vehicles, embedded microprocessor and sensors in the road side devices, and the use of identical digital maps in the whole network. One of the most important features is that there is no limit in the batteries lifetime of the road side devices or in the storage size as well. Some research results in network devices and sensors nodes for this kind of networks are proposed in [15] where the tasks of the mobile sensors, the traffic control in the system, the content of the shared information and the communication protocols are described. Another study [17] focused on providing a safer road presents a cost-effective roadto-car (R2C) approach based on WSNs. It is based on the implementation of several sensor devices along the road, which is divided into road segments. An island of sensors will gather information about the weather status as well as other information from the vehicles. This study gives a new approach which can be used in two different services: accident prevention and post-accident investigation. Such information can be used to save lives and also to be used by the forensic teams as a reliable source of the facts. Also, [18] presents the benefit of using multiple access technologies and multiple radios in a collaborative manner, to create an advanced heterogeneous vehicular network (AHVN) architecture.

\section{BASICS OF A HSVN FRAMEWORK}

The main feature offered by vehicular networks is the capability to distribute traffic road information among the vehicles of a road. Nodes in vehicular networks will be able to access to different information related to their environment. This information can be useful to assist data routing protocols in VANETs to make proper decisions, e.g. detect those vehicles with similar routes to compose routing paths seeking to increase path lifetimes. The flooding mechanism is not very functional because of the potential high number of nodes in a VANET; instead, the multihop network feature is used, which allows spreading information vehicle by vehicle until the destination node is reached, in case sender and destination are not in the same transmission range. A dissemination protocol to distribute road data through the VANET is required. 
Public transportation may also be involved in the communication network. Buses can operate as reliable VANET nodes and also to offer Internet connection to other passing vehicles. In VANETs, it is typically assumed that each node in the network is equipped with some navigation system and also with a GPS. Nowadays, most of the modern cars already have a GPS installed, so that car navigation systems (e.g. Tom-Tom [20], GARMIN [21]) are able to know their geographical position and to obtain digital maps as well. This information can be useful for the car navigation system to make proper decisions, for example to take the most reliable and safer noncongested road from several options.

A simple, fast and efficient communication protocol has to be designed to allow communication between VANETs and WSNs. Both networks must share data that carry road information and short video streams. The data interchange has to be produced very fast, since the interval in which the vehicle is under the transmission range of the WSNs is very short. The cooperation between WSNs and VANETs makes it possible to extend the transmission range in a VANET to a larger region with the cooperation among ad hoc nodes within both networks. Vehicles can store road information in the WSN's sink as they pass through that WSN. Later on, other passing cars can recover that information from the WSN's sink, as it can be seen from Figure 1.

The content of the interchanged messages regarding road safety has to be defined. Messages have to include information (e.g. weather conditions, location of accidents, possible building work, etc) about different road segments. Messages can include a low quality image of the incoming intersections, so that the co-pilot or the driver could have a quick look to foresee actual information of the road ahead. After that, the data interchanged between the WSN's gateway and the vehicle in the VANET, will be stored or updated at their respective data bases. Moreover, the protocol to be designed has to manage other kind of data interchange (see Figure 1) which is described as follows:

- Group leader vehicles (cars A and C in Figure 1) gather road information from the vehicles behind in their respective groups.

- The road information is interchanged between group leaders which travel in opposite directions (cars A and $\mathrm{C}$ in Figure 1).

- The new received information is spread from the group leader among the vehicles in the group.

- Passengers can access to the Internet by means of the APs spread along the roadside or also by means of public transportation with Internet connection.

Another important feature to mention is that the configurable parameters of the framework might adapt dynamically according to the network's state (e.g. amount of car traffic, packet losses, and packet delays). As it is pointed out in the future work section, in a future work we will design a HSVN routing protocol based on a cross-layer scheme, so that different protocol layer levels can collaborate to improve the performance. In addition, the routing protocol will be able to adapt and reconfigure its parameters, taking into account representative protocol level parameters such as the video quality perceived by users (application level), packet losses (network level) and retransmissions (MAC level). Also, the framework should be able to offer QoS to provide multimedia information (e.g. video-streaming) regarding accidents or traffic density, as well as data carrying road information (e.g. weather, road congestion).

\subsection{Mobility models in VANETs}

In a vehicular scenario, cars do not move freely throughout the whole area. Instead, they follow the lanes of the road or the streets in a city, they follow traffic signals and they are aware of the other vehicles. In addition, the node's distribution is not constant; on the contrary vehicles tend to create groups. The type of road must also be considered because most of the parameters (number of lanes, speed of the nodes, etc.) change from one scenario to another (rural, motorway, urban). In order to achieve realistic results, all these features have to be taken into account when designing a HSVN framework.

A mobility model describes the movement pattern followed by the nodes in a specific scenario. Those mobility models need to be included in the simulations carried out to analyse the performance evaluation of new routing and communication protocols designed for HSVNs. Choosing the proper simulation settings and mobility model is crucial since these configuration parameters will determine the results. For instance, in an urban scenario it is very important to include obstacles, roads, traffic lights and signals. Some research works, such as [22], show how important it is to consider a realistic mobility model for VANETs to obtain reliable simulated results close to real scenarios. In that work, [22], the authors carried out several simulations with several models like Freeway [23], Manhattan [23], CSM (City Section Mobility) [24], SSM (Stop Sign Model) [25], TSM (Traffic Sign Model) [25], STRAW [26], etc. They conclude that the first three are inappropriate for simulating VANETs, whereas the last three ones are more realistic, as they involve traffic lights and stops signs.

\subsection{Proposal of a communication protocol between WSNs and VANETs}

In the following, we describe the communication algorithm between WSNs and VANETs that we have designed. The main purpose is to fulfil all the types of communications produced between vehicles and static road sensors.

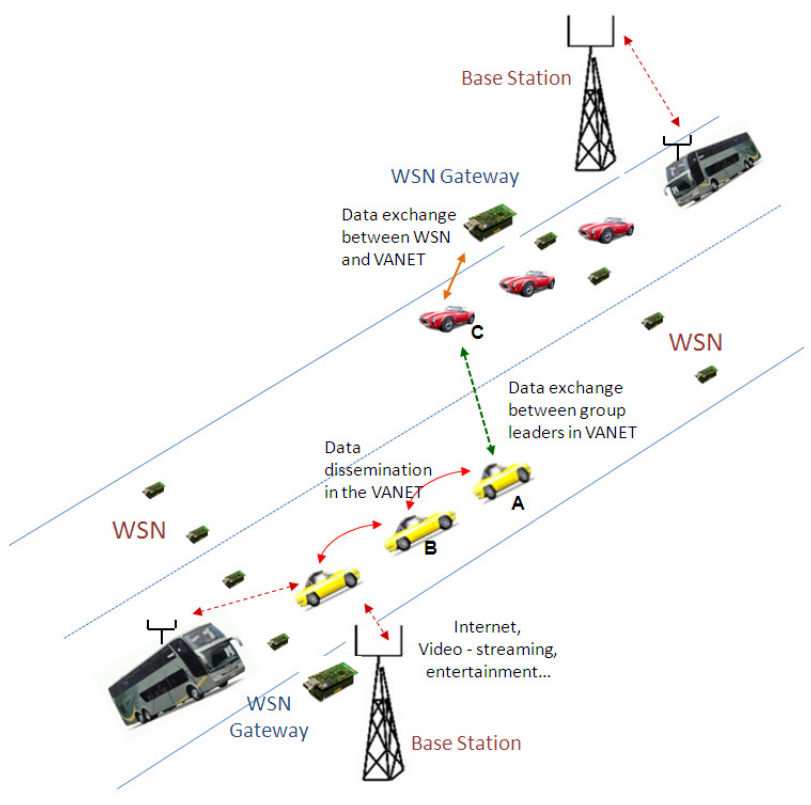

Figure 1. General scenario of a HSVN. 
There are three types of communications to be considered between WSNs and VANETs, which are depicted in Figure 1:

a) Communications between static sensors in a WSN and vehicles in a VANET

$\mathrm{WSN} \rightarrow$ Vehicle:

a.1. The WSN's gateway detects a vehicle within its transmission range.

a.2. The WSN's gateway sends a connection request (14 bytes) to the passing vehicle.

Vehicle $\rightarrow$ WSN:

a.3. The vehicle sends back an ACK (14 bytes) to the WSN's gateway. This ACK contains the coordinates of its destination (20 bits). This way the vehicle sets high priority to the information of its own interest. It also includes the ID (identification, 20 bits) of the vehicle.

$\mathrm{WSN} \rightarrow$ Vehicle:

a.4. Transmission of a packet including road information regarding all segments that are in the path along the destination of the passing vehicle. Also, data regarding other paths. The packet contains this information:

a.4.1. A two-bits header field per road segment that includes information about the state of the traffic density of each road segment along the path. The data codification is: $0=$ free segment, $1=$ semicongested segment, $2=$ very congested segment, $\mathrm{n} / \mathrm{i}=$ unknown information.

a.4.2. A two-bits header field per road segment that includes information about the weather/accident state of the roads. The data codification is: $0=$ good weather conditions, $1=$ ice, $2=$ rain, $3=$ accident.

a.5. Transmission of a small image $(50$ Kbytes approx.) regarding the next cross of the path in the vehicles' destination. After that, other images regarding other intersections can be sent, as long as both nodes are in transmission range.

Vehicle $\rightarrow$ WSN:

a.6. The vehicle sends to the WSN's gateway road information (data and image) which was previously gathered from other vehicles or other WSN. The WSN updates that information (if newer) regarding the state of the roads. The content and codification of the packets that include this road information, is as described in a.4.

Vehicle $\rightarrow$ WSN:

a.7. The vehicle reaches the coverage limit and the connection ends.

b) Vehicle-to-Vehicle communications. Vehicles move in the same direction

Vehicle A $\rightarrow$ Vehicle B:

b.1. Vehicle B is detected by vehicle A within its transmission range. Vehicle $\mathrm{B}$ is behind vehicle $\mathrm{A}$ in the same direction (see Fig. 1).

b.2. A connection request (14 bytes) is sent by vehicle A to vehicle $B$.
Vehicle B $\rightarrow$ Vehicle A:

b.3. An ACK (14 bytes) is sent by vehicle B to vehicle A. It includes its ID (identification, 20 bits). It also includes its destination coordinates.

Vehicle A $\rightarrow$ Vehicle B:

b.4. Data transmission regarding the state of the roads and traffic density of all the road segments that are in the destination path of vehicle $\mathrm{B}$ and also of the roads within other paths. The packet contains this information:

b.4.1 A two-bits header field per road segment that includes information about the state of the traffic density of the road segment. The data codification is: $0=$ free segment, $1=$ semicongested segment, $2=$ very congested segment, $\mathrm{n} / \mathrm{i}=$ unknown information.

b.4.2 A two-bits header field per road segment that includes information about the weather/accident state of the roads. The data codification is: $0=$ good weather conditions, $1=$ ice, $2=$ rain, $3=$ accident.

b.5. Transmission of a small image (50 Kbytes approx.) regarding the next cross of the path in the destination of vehicle B. After that, other images regarding other intersections can be sent, as long as both nodes are in transmission range.

Vehicle A $\rightarrow$ Vehicle B:

b. 6 Vehicle A reaches the coverage limit of vehicle B and the connection ends.

This way, the road information is spread backward through the group of vehicles. In addition, every new incoming vehicle in the group sends its own new road information forward till the leader, through the other relying vehicles in the group. This way, the leader gathers the whole road information of its group of vehicles.

c) Vehicle-to-Vehicle communications. Vehicles move in opposite direction

Vehicle $\mathrm{A}$ in one direction $\rightarrow$ Vehicle $\mathrm{C}$ in the opposite direction (see Figure 1).

c.1. In case that the detected vehicle goes in opposite direction, the connection will only be established between the first vehicles of both groups (group leaders). The communication between vehicle $\mathrm{A}$ and vehicle $\mathrm{C}$ is as described in $\mathrm{b}$ ) from b. 1 to b.6.

c.2. Each group leader disseminates the new road information among the vehicles within its own group.

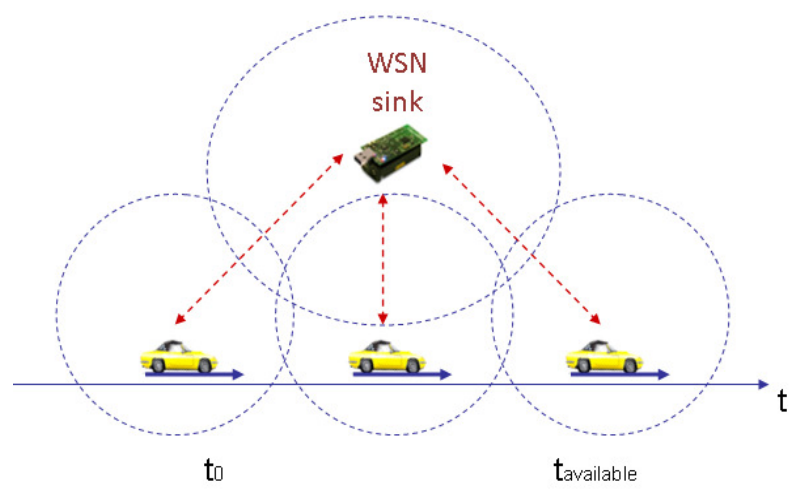

Figure 2. Temporal available time to interchange messages 
Regarding the amount of information needed to communicate the state of the whole road segments that belong to a destination, let us make some considerations. There may be a different number of road segments per destination. Basically, it depends on the type of scenario (urban, rural, motorway) and on the total road length to that destination. For example, in case of a motorway with a total $500 \mathrm{~km}$ of an average long trip and road segments (located between exits) of $10 \mathrm{~km}$ each, there would be about 50 segments in such a trip. In case of a rural road with an average $50 \mathrm{~km}$ trip and road segments of $5 \mathrm{~km}$, there would be around 10 road segments. Finally, in city scenarios where typical trips take around $5 \mathrm{~km}$ and road segments measure about $200 \mathrm{~m}$, there are around 25 segments per average trip. Next list summarizes the number of road segments per typical trip in each scenario.

- In a motorway, 50 road segments, $500 \mathrm{~km}$ trip

- In rural road, 10 road segments, $50 \mathrm{~km}$ trip

- In city, for 25 road segments, $5 \mathrm{~km}$ trip

In order to estimate if there is enough time to fulfil the data interchange between the WSN sink and a passing vehicle, let us see a numerical example. Let us consider that packets take 1000 bytes, then it will be possible to code up to 1995 road segments using a single packet:

$\frac{1000 * 8(\text { bits })-20(\text { ID bits })}{4(\text { ID header bits to code a road segments })}=1995$ road segments

A typical interchange of data between the WSN's gateway and a car in the VANET includes information about 50 road segments (in case of a motorway trip) corresponding to the information of interest of that vehicle. In addition, there is additional room in a single packet to include road information about 40 other trips:

$\frac{1995 \mathrm{road} \mathrm{segm} .-50 \mathrm{road} \text { segm.in the trip of interest }}{50 \mathrm{road} \mathrm{segm.} / \text { trip }}=40$ trips

Equivalently, in a rural scenario, a single packet can include road information of up to 200 trips or destinations, i.e. 1995/10. In a city scenario, a single packet can include road information of up to 79 destinations, i.e. 1995/25. Each vehicle transmits a beacon every $10 \mathrm{~ms}$ so that other ad hoc nodes are aware of them. When the car is out of range of the WSN sink node, its beacons are not received any more and therefore the sink node stops sending road information to that vehicle. The IEEE 802.11b MAC has a nominal link bandwidth of $11 \mathrm{Mbps}$ and a throughput around $t h=7 \mathrm{Mbps}$ (for UDP like traffic). We consider a maximum speed of the vehicle of $v \mathrm{~km} / \mathrm{h}$ and a transmission range of the WSN sink node of $r \mathrm{~m}$. Then, the available time for the data interchange is $t_{\text {available }} \approx 2 r / v$ sec, as depicted in Fig. 2. During this time the communication is established and the transmission of all the packets must be done, according to the messages interchange described in point a) of the communication protocol.

The data information regarding all the road segments is transmitted in a single packet of size $p=1000$ bytes, which takes $t_{\text {road segments }}=p / t h=1,14 \mathrm{~ms}$. The transmission of an image of 50 Kbytes takes 50 packets and $t_{\text {image }}=0,057 \mathrm{sec}$. The total time required to send that information is $t_{\text {required }}$ $=t_{\text {road_segments }}+t_{\text {image }}=0,058 \mathrm{sec}$. Thus, if $t_{\text {required }}<t_{\text {available }}$ the exchange of information (data and images) between the car and the sensor sink can be successfully done. Furthermore, more images regarding other intersections could be sent as well within the available interval of time, $t_{\text {available }}$.
Let us consider a transmission range of the sink WSN node of $r=80 \mathrm{~m}$. Let us estimate the $t_{\text {available }}=2 r / v$ values for each type of scenario.

- In a motorway, for $v=120 \mathrm{~km} / \mathrm{h}, t_{\text {available }}=4,8 \mathrm{sec}$

- In a rural road, for $v=80 \mathrm{~km} / \mathrm{h}, t_{\text {available }}=7,2 \mathrm{sec}$

- In a city, for $v=50 \mathrm{~km} / \mathrm{h}, t_{\text {available }}=11,52 \mathrm{sec}$

We can see that $t_{\text {required }}=0,058 \mathrm{sec} .<<t_{\text {available }}$ in every scenario, so that more additional images $\left(t_{\text {image }}=0,057 \mathrm{sec}\right)$ could be sent during the available period of time.

\section{SIMULATION RESULTS}

To analyse the performance of the proposed communication protocol between WSNs and VANETs, we have carried out several simulations of data transmissions between different nodes in a HSVN. We have used the freeware simulator NCTuns6.0 (National Chiao Tung University Network Simulator) [20]. The VANET consists of four mobile nodes which have the CarAgent mobility model that allows nodes to follow roads, be aware of other vehicles and of traffic signals and traffic lights. Vehicles receive packets from the fixed sink node in the WSN. In this work, we will analyse a part of the general design of the framework, which is shown in Fig. 1. As a starting point, we will evaluate the performance of our communications protocol between WSN and VANETs under two well-known routing protocols for ad hoc networks, AODV (Ad hoc On Demand Distance Vector) [27] and DSR (Dynamic Source Routing) [28]. Fig. 3 depicts the scenario, where we can see a group of four vehicles that move in the same direction, and they pass a WSN sink. There are traffic lights located in the corners of the road, so that cars reduce their speed as they approach the traffic lights and they stop there if it is a red light.

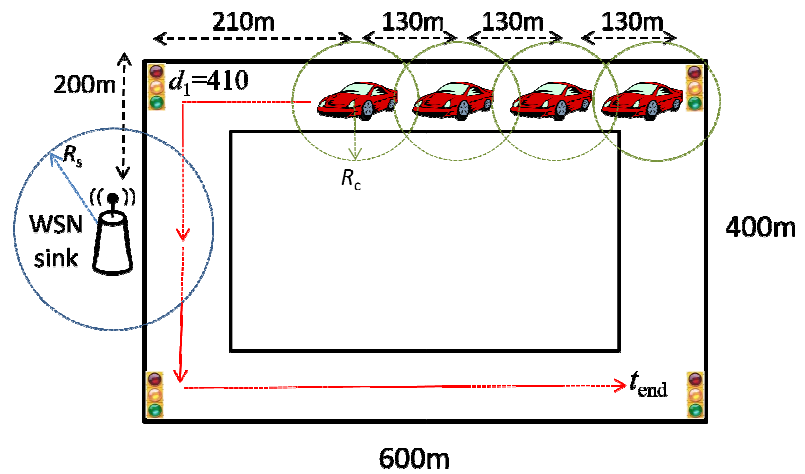

Figure 3. Scenario under evaluation with NCTUns.

In this simple scenario, we assume that the WSN sink previously gathered the road segment information monitored by the sensors within its WSN. In the simulations, the WSN sink sends packets that carry the data of the road segments until the last car of the group. Therefore, a forwarding route is composed by using the other cars, who also receive such road information. The cars are spaced $d=130 \mathrm{~m}$. The distance from the first car of the group to the sink is $d_{1}=410 \mathrm{~m}$. Assuming an average speed of the cars of $\mu \mathrm{m} / \mathrm{s}$, the moment $t_{1}$ in which the first car arrives to the sink is:

$$
t_{1}=\frac{d_{1}-R_{s}}{\mu}
$$

Whereas the last moment $t_{2}$ in which the last car is out of coverage of the sink is:

$$
t_{2}=\frac{d_{1}+3 \cdot d+R_{s}}{\mu}
$$


According to equations (3) and (4), Table 1 summarizes the ratio of the time during which the communication can be produced, since the moment when the routing protocol can find an available path. The simulation time lasts $t_{\mathrm{end}}=80 \mathrm{sec}$.

We have modified the speed of the nodes and the size of the packets. We have analysed the performance losses under two routing protocols. Table 2 shows the configuration parameters in the different simulations. We have compared the performance of AODV to DSR as routing protocols in terms of packet losses and average end-to-end delay. In Fig. 4, the evolution of the packet losses using AODV is shown. We show the $80 \%$ confidence interval for these values, where five simulations per point have been carried out.

Table 1. Ratio of time when there is connectivity.

\begin{tabular}{|lllll|}
\hline$\mu(\mathrm{m} / \mathrm{s})$ & $t_{1}(\mathrm{sec})$ & $t_{2}(\mathrm{~s})$ & $t_{2}-t_{1}(\mathrm{~s})$ & $\left(t_{2}-t_{1}\right) / t_{\text {end }}$ \\
\hline 40 & 18,9 & 90 & 71,1 & $88 \%$ \\
60 & 12,6 & 60 & 47,4 & $59 \%$ \\
80 & 9,45 & 45 & 35,5 & $44 \%$ \\
100 & 7,56 & 36 & 28,4 & $35 \%$ \\
120 & 6,3 & 30 & 23,7 & $29 \%$ \\
\hline
\end{tabular}

Table 2. Simulation settings of the HSVN.

\begin{tabular}{|l|l|}
\hline Average speed of the nodes & $\mu=40$ to $120 \mathrm{~km} / \mathrm{h}$ \\
\hline Number of road lanes & 4 (two in each direction) \\
\hline Road length & $2 \mathrm{Km}$ \\
\hline $\begin{array}{l}\text { Number of Mobile nodes in the } \\
\text { VANET }\end{array}$ & 4 vehicles \\
\hline Number of nodes in the WSN & 1 sink node \\
\hline $\begin{array}{l}\text { Transmission Range of the } \\
\text { nodes (WSN and VANET) }\end{array}$ & $R_{s}=R_{c}=200 \mathrm{~m}$ \\
\hline Routing protocol in the HSVN & AODV, DSR \\
\hline Packet size & $500,1000,1500$ bytes \\
\hline Time of simulation & $t_{\text {end }}=80 \mathrm{sec}$. \\
\hline Data source rate (CBR) & $1 \mathrm{Mbps}$ \\
\hline MAC & IEEE $802.11 \mathrm{~b}$ \\
\hline Nominal capacity & $11 \mathrm{Mbps}$ \\
\hline
\end{tabular}

Figure 4 shows the percentage of packet losses for AODV, which produces higher losses for higher speeds. The reason is that vehicles behind another vehicle in the VANET scenario must adapt their speed to the speed of that car in front of them, according to the CarAgent mobility model implemented in NCTUns. That is, the first car can go faster than the others behind, so that when the link to the second car behind breaks an alternative route must be found. This is especially noticeable for high speeds, whereas for low speeds cars tend to remain in group so that the links last longer, which produces lower losses.

Figure 5 shows the percentage of packet losses for DSR. In general, DSR shows lower losses than AODV in this scenario. In the same way, DSR shows higher throughput than AODV as it can be seen in Fig. 6. This is due to the fact that in this scenario AODV uses longer paths during more time than DSR. When the first 3-hop path breaks (the first link between the first and second cars breaks), AODV spends less time in finding a new path (a 2-hop path) than DSR. When the first 3-hop route breaks, DSR spends more time than AODV to find an alternative route, and finally when DSR finds an alternative path, the cars are closer to the sink. Even DSR usually finds the shortest 1-hop route to the final car in the queue, i.e. the destination. That is, the 2-hop is almost not being used by DSR, specially for higher speeds, where cars arrive faster to the transmission range of the sink. This produces lower losses for
DSR in high speeds. Conversely, AODV finds an alternative route sooner, which is the 2-hop route that incurs in higher chance of collisions than the single hop route.

Figure 6 shows the throughput for AODV and DSR. Continuous lines show results for AODV whereas dotted lines show results of DSR. The two square-shaped lines (in the bottom of Figure 6) correspond to 500 bytes packets size, the two triangle-shaped (in the middle) corresponds to 1500 bytes. Finally, the two circle-shaped lines (at the top) correspond to 1000 bytes of packet size. It can be seen that DSR performs better. Also, we can observe that for a 1000 bytes packet the results are in general better.

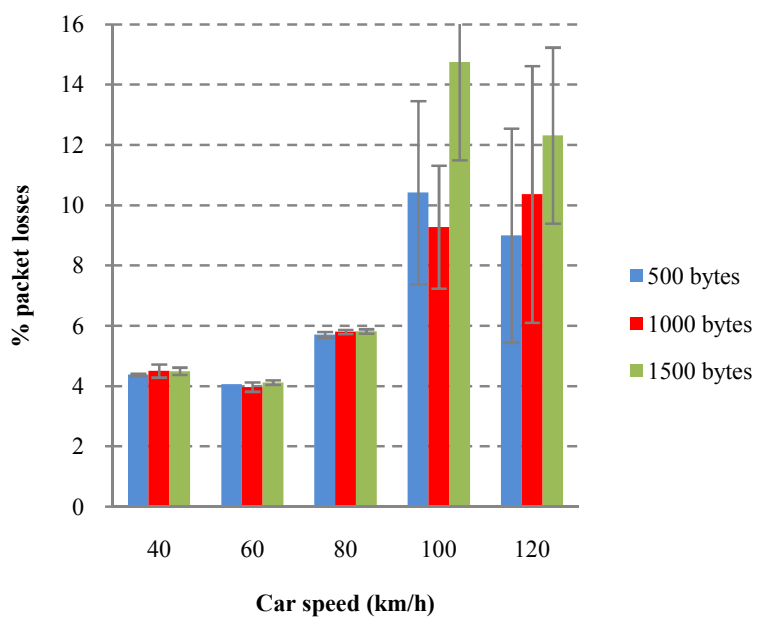

Figure 4. Packet losses evolution for AODV.

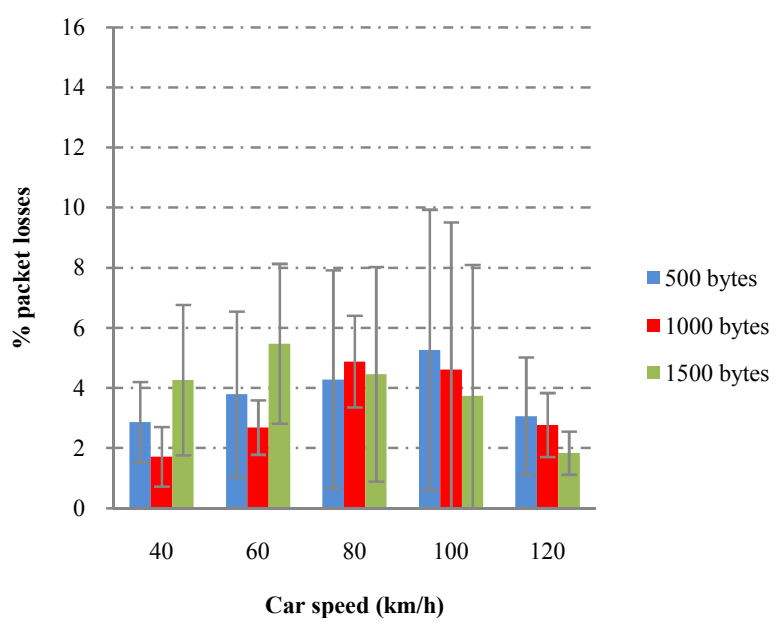

Figure 5. Packet losses evolution for DSR.

Results for average packet delays are shown in Figures 7 and 8. It can be seen that end-to-end delays are slightly higher using AODV than using DSR. For low speeds the delay is around 1-2 sec for DSR and around 2-3 sec for AODV, whereas for high speeds the delay is around 1-3 sec for DSR and around 4-7 sec for AODV. As it has been said above in the analysis done for the losses in Figures 4 and 5, due to the fact that in this scenario AODV uses the longer 3-hop and 2-hop paths during more time than DSR, the packet end-to-end delays for AODV are also longer than for DSR. That is, after the breakage of the first 3hop path, AODV finds sooner the 2-hop path, whereas DSR takes longer to find an alternative path (it has no alternative 
routes in cache) and even it can find the direct 1-hop route. This shorter route will produce that the end-to-end packet delay for DSR reduces.

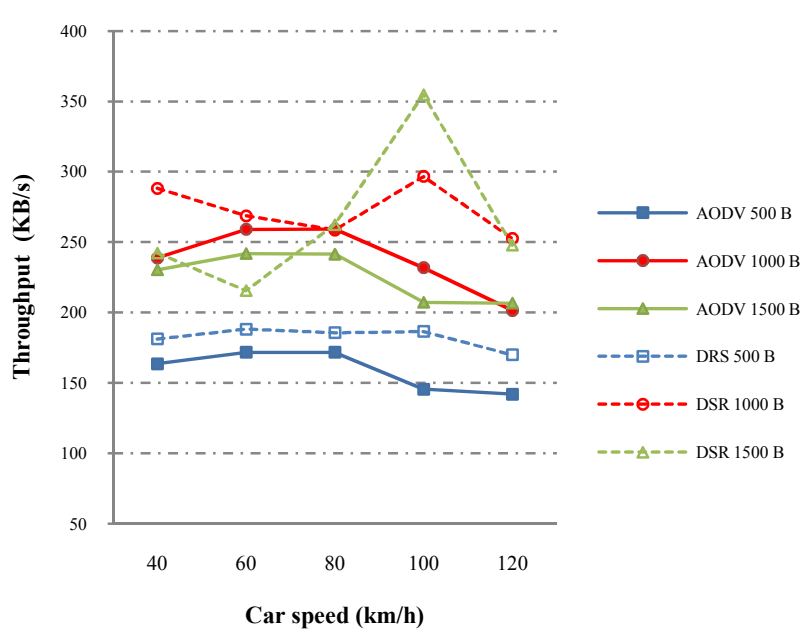

Figure 6. Throughput with AODV and DSR.

Let us briefly review the process to establish new routes followed by both routing protocols. According to the RFC for DSR [28] the routes timeout in cache was set to 300 s, so they remain in cache during the whole simulation time (80s). After route breakage, DSR first searches for an alternative route in cache and if there is no other route to destination, a new route discovery process starts. Notice that in this scenario there seems to be just one route that includes all the cars from the sink to the last car of the group. However, DSR may find more than one route in case of having several cars in transmission range, which only happens when they get closer enough to the sink. In this case, several cars are under transmission range of the sink so that several routes to destination can be found. After route breakage, DSR can quickly use a route stored in cache in case of having another, otherwise DSR has to find a new route. This happens when the first 3-hop route breaks and there is no alternative route in cache. Instead of using a cache, AODV maintains a simple 1-hop routing table in every node [27], so that this partial knowledge of topology helps the routing mechanism to find promptly an alternative route after breakage.

Regarding the packet size, AODV shows best results for high speeds when the packet size equals 500 bytes, whereas for lower speeds the packet size has minor effect. For DSR lower packet sizes are preferable under lowers speeds and higher packet sizes are preferable under higher speeds.

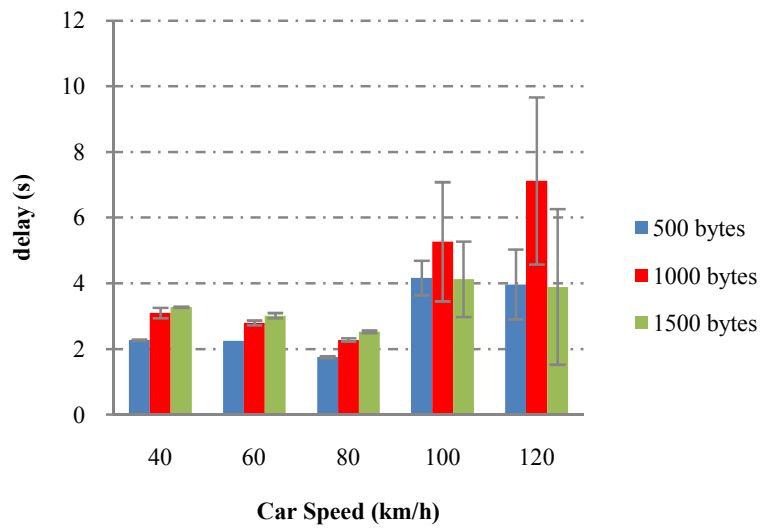

Figure 7. End-to-end packet delay for AODV. According to the results for this scenario it can be seen that DSR performs better than AODV in terms of losses and throughput, especially in case of higher speeds (higher than 80 $\mathrm{km} / \mathrm{h}$ ). On the other hand, DSR also performs better than AODV in terms of delay for every car speed.

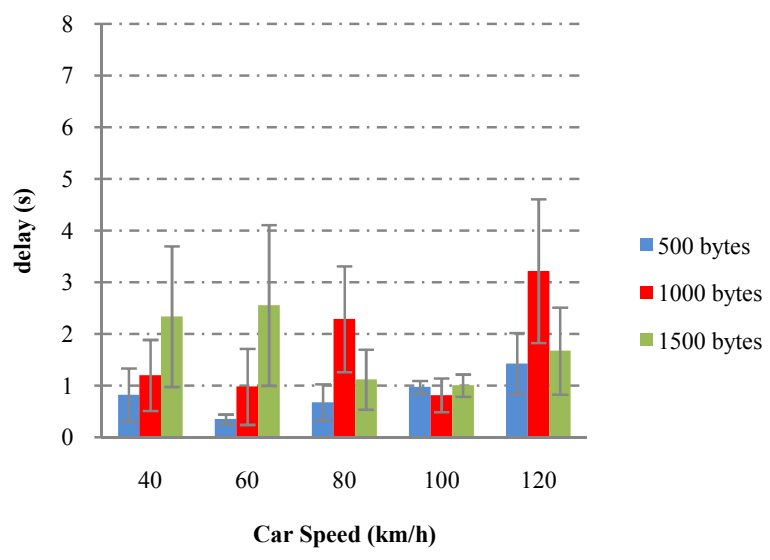

Figure 8. End-to-end packet delay for DSR.

\section{CONCLUSIONS AND FUTURE WORK}

In this article we have shown the performance of the routing protocols AODV and DSR in a HSVN framework that includes a proposal for a communications protocol between WSNs and VANETs. Simulation results for the scenario under evaluation, show the effectiveness of DSR compared to AODV regarding end-to-end delays, losses and throughput.

In an immediate work we will modify the routing protocol to include some additional features suitable for vehicular networks, such as an algorithm that considers location and speed of the vehicles (obtained by GPS in real vehicular networks) to compose proper paths (those that may remain longer) and to switch to an alternative path prior to actual breakage. Besides, analytical models to compute the available bandwidth of the paths will be designed to assist the routing protocol. Furthermore, other aspects will be tackled to improve the routing in HSVN, such as considering the different type of traffics (e.g. warnings, road messages, video-streaming, Internet browsing) and the different QoS requirements according to the type of traffic, along with the different interface technologies that can include the vehicles (i.e. WIFI, 3G, satellite), so that high-priority warning messages will be sent through the best available connection, while video-streaming services can use another technology.

As future work we will analyse the performance of the system under other routing protocols more specific for VANETs, e.g. GSR (Geographic Source Routing) [29], SAR (Spatial Aware Routing) [30], and VADD (Vehicular Assisted Data Delivery) [31]. In addition, we will evaluate the system performance using the MAC IEEE $802.11 \mathrm{p}$ specification, which is focused on VANETs. Also we will design a QoS-aware cross-layer routing protocol for VANETs and we will compare its performance to AODV, DSR and other VANET routing protocols. 


\section{ACKNOWLEDGMENTS}

This work has been funded by the Spanish Ministry of Science and Education under the project ITACA (TSI2007-65393-C0202 ) and partially supported by the Comissionat per a Universitats $i$ Recerca del DIUE from the Generalitat de Catalunya and the Social European Budget (Fons Social Europeu) with the grant FPI-AGAUR and by the Autonomous University of Sinaloa, Mexico.

\section{REFERENCES}

[1] CVIS. Cooperative Vehicular-Infrastructure Systems. http://www.cvisproject.org/

[2] COME. Co-operative Intelligent Road Transport Systems: Vehicle-to-Vehicle and Vehicle-toInfrastructure communications. [Online] http://www.comesafety.org/.

[3] NCTUns 6.0 (Network Simulator and Emulator). [Online] 2010. http://nsl.csie.nctu.edu.tw/nctuns.html.

[4] C. Siva Ram Murthy, B.S. Manoj. "Ad Hoc Wireless Networks: Architectures and Protocols". June 3, 2004 ISBN: 978-0131470231.

[5] K. Sohraby, D. Minoli, T. Znati. "Wireless Sensor Networks: Technology, protocols and applications". Wiley Interscience, 2007. ISBN: 978-0-471-74300-2.

[6] H. Hartenstein, K. Laberteaux. "VANET Vehicular Applications and Inter-Networking Technologies (Intelligent Transportation Systems)". Wiley, March 2010. ISBN: 978-0470740569.

[7] S. Olariu, M. Weigle. "Vehicular Networks from Theory to Practice". Norfolk Virginia, USA. Chapman and Hall, 2009. ISBN: 978-1420085884.

[8] D. Jiang, L. Delgrossi. "IEEE 802.11p: Towards an International Standard for Wireless Access in Vehicular Environment". 67th IEEE Vehicular Technology Conference VTC. Singapore, 2008.

[9] IEEE P802.11p/D3.0. "Draft Amendment to Standard for Information Technology-Telecommunications and Information Exchange between Systems-Local and Metropolitan Area Networks-Specific Requirements Part 11: Wireless LAN Medium Access Control (MAC) and Physical Layer (PHY) SpecificationsAmendment 7: Wireless Access in Vehicular Environment". 2007.

[10] C2C. Car-To-Car Communication Consortium. [Online] http://car-to-car.org/.

[11] CARLINK. Wireless Traffic Service Platform for Linking Cars. [Online] http://carlink.lcc.uma.es/.

[12] eSafety Forum. [Online] http://www.esafetysupport.org/

[13] INFOTRANSIT. Reial Automòbil Club de Catalunya (RACC). [Online] http://infotransit.es.

[14] Google Maps. [Online] http://maps.google.com/.

[15] F. Kong, J. Tan. "A Collaboration-based Hybrid Vehicular Sensor Network Architecture". IEEE International Conference on Information and Automation. Zhangiajie, China, 2008.

[16] E. Weingärtner, F. Kargl. "A Prototype Study on Hybrid Sensor Vehicular Networks". 6 KuVS Fachgespräch Sensornetzwerke, RWTH-Aachen Technical Report, no. AIB 2007-1, Aachen, Germany. 2007.
[17] J. Bohli, O. Ugus, D. Westhoff. "A Secure and Resilient WSN Roadside Architecture for Intelligent Transport System". ACM Workshop on Wireless Security. Alexandria, Virginia, USA, 2008.

[18] E. Hossain, G. Chow, V. Leung, R. McLeod, J. Mišic, V. Wong, O. Yang. "Vehicular telematics over heterogeneous wireless networks: A survey. Computer Communications”. pp. 775-793. Elsevier 2010.

[19] B. Hofman-Wellenhof, H. Lichtenegger, J. Collins. "Global Positioning System: Theory and Practice". Springer, 2001. 5th Ed. ISBN: 3211835342.

[20] TomTom. [Online] http://www.tomtom.com/.

[21] GARMIN.[Online] http://www.garmin.com/garmin/cms/site/es.

[22] D. Djenouri, E. Nekka, W. Soualhi. "Simulation of Mobility Models in Vehicular Ad hoc Networks". 1st ICST Int. Conf. On Ambient Media and Systems (Ambi-sys). Quebec, Canada, 2008. ISBN: 9789639799165.

[23] F. Bai, N. Sadagopan, A. Helmy. "Important: a framework to systematically analyse the impact of mobility on performance of routing protocols for ad hoc networks". The 22th IEEE Annual Joint Conference on Computer Communications and Networking INFOCOM'03. pp. 825-835. 2003.

[24] V. Davies. "Evaluating mobility models within an ad hoc network". Colorado School of Mines, Colorado, USA, Tech. Rep. Master's thesis, 2000.

[25] A. Mahajan, N. Potnis, K. Gopalan, A. I. A. Wang. "Urban mobility models for vanets". Proceedings of the 2nd IEEE International Workshop on Next Generation Wireless Networks. December 2006.

[26] D. R. Choffnes, F. E. Bustamante. "An integrated mobility and traffic model for vehicular wireless networks". 2nd ACM international workshop on Vehicular ad hoc networks, VANET'05. ACM Press. pp. 69-78. 2005.

[27] Ad hoc On-Demand Distance Vector (AODV) Routing. http://www.ietf.org/rfc/rfc3561.txt

[28] The Dynamic Source Routing Protocol (DSR. http://www.rfc-editor.org/rfc/rfc4728.txt

[29] C. Lochert, H. Hartenstein, J. Tian, H. Füßler, D. Hermann, M. Mauve. "A Routing Strategy for Vehicular Ad Hoc Networks in City". IEEE Intelligent Vehicles Symposium '03, pp. 156-161. 2003.

[30] J. Tian, L. Han,K. Rothermel. "Spatially Aware Packet Routing for Mobile Ad Hoc Inter-Vehicle Radio Networks". IEEE Intelligent Transportation Systems, Shanghai, pp. 1546-1551. China, 2003.

[31] J. Zhao, J. Cao. "VADD: Vehicle-assisted data delivery in vehicular ad hoc networks". 25th IEEE International Conference on Computer Communications, pp.1-12. INFOCOM 2006. 